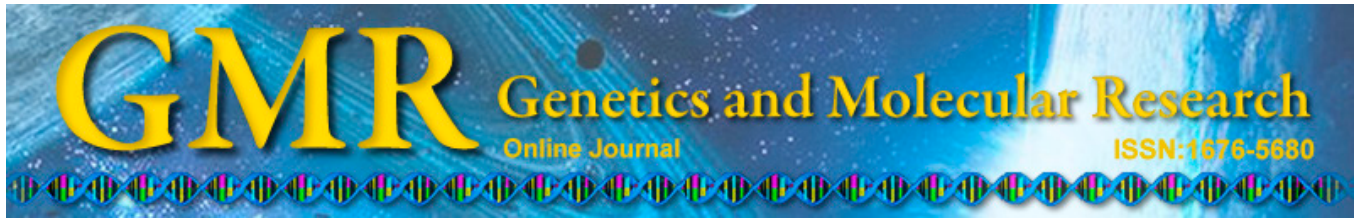

\title{
A new repertoire of informations about the quorum sensing system in Salmonella enterica serovar Enteritidis PT4
}

\author{
M.E.M. Campos-Galvão' ${ }^{1}$ T.D.S. Leite ${ }^{1}$, A.O.B. Ribon ${ }^{2}$, E.F. Araújo ${ }^{1}$ and \\ M.C.D. Vanetti ${ }^{1}$ \\ ${ }^{1}$ Departamento de Microbiologia, Universidade Federal de Viçosa, \\ Viçosa, MG, Brasil \\ ${ }^{2}$ Departamento de Bioquímica, Universidade Federal de Viçosa, \\ Viçosa, MG, Brasil \\ Corresponding author: M.C.D. Vanetti \\ E-mail: mvanetti@ufv.br
}

Genet. Mol. Res. 14 (2): 4068-4084 (2015)

Received July 25, 2014

Accepted December 2, 2014

Published April 27, 2015

DOI http://dx.doi.org/10.4238/2015.April.27.22

\begin{abstract}
Salmonella spp are among the main causative agents of foodborne diseases. Some phenotypes associated with increased drug resistance and virulence are regulated by quorum sensing (QS). In the present study, the autoinducer (AI)-1- and -2-mediated QS mechanisms were characterized in Salmonella enterica serovar Enteritidis PT4 for the first time. Salmonella Enteritidis did not produce AI-1. Phylogenetic analysis of nucleotides encoding the SdiA protein, the response regulator of AI-1-mediated QS, and comparative alignment of its amino acids showed that the gene and protein are conserved within the same bacterial genus. Thus, bacteria of the same genus respond to the same AIs. However, this finding did not preclude the possibility that Salmonella Enteritidis might respond to AIs released from bacteria of a different genus, which might confer a competitive advantage to this pathogen. We found that the regulation of AI-2-mediated QS in Salmonella Enteritidis is similar to that in serovar Typhimurium. The elucidation of
\end{abstract}


the AI-1- and AI-2-mediated QS mechanisms in Salmonella Enteritidis will contribute to the development of new control strategies for this pathogen by indicating new targets for antimicrobial drugs.

Key words: Quorum sensing; Salmonella Enteritidis PT4; SdiA

\section{INTRODUCTION}

Salmonella spp are the causative agents of a wide variety of diseases in humans and animals, including enteric fever and gastroenteritis (De Oliveira et al., 2014). The most frequent infections caused by eating food contaminated with $S$. result in gastroenteritis and are attributed to Salmonella enterica serovars Typhimurium and Enteritidis (Ahmer and Gunn, 2011; De Oliveira et al., 2014). The Salmonella enterica serotype Enteritidis is the most common cause of foodborne disease worldwide. This serovar is closely associated with consumption of eggs, poultry, and pork (Maciel et al., 2011).

The economic losses caused by Salmonella spp infections have attracted increasing attention in developed countries, particularly the United States, Canada, and European countries, although their impact may be much higher in developing countries, where little information is available. The financial costs are not only related to research, prevention, and treatment of human diseases but also to the maintenance of adequate sanitary conditions throughout the food production chain, and they affect both the public and private sectors (Sockett, 1991). Therefore, scientific research contributes to a solution to the problem by investigating pathogenic mechanisms and pathogen control.

A cell density-dependent signaling system was first described by Nealson and Hastings (1979), who reported the bioluminescent phenotype produced by Vibrio fischeri. Later, this quorum-dependent cellular communication mechanism, or quorum sensing (QS), was characterized in many other genera of bacteria. With this system, bacterial cells are able to monitor the presence of other bacteria surrounding them based on the production and response to low-molecularmass signaling molecules termed autoinducers (AIs) (Fuqua et al., 1994; Kalia, 2013).

QS-mediated intracellular communication is used for regulating a wide variety of processes in bacterial communities. These include biofilm formation, resistance to antibiotics, induction of virulence factors, cell differentiation, nutrient flow, and collective defense against other competing microorganisms or against eukaryotic host defense mechanisms (Miller and Bassler, 2001; March and Bentley, 2004; Kalia, 2013). Furthermore, QS optimizes population survival through regulating differentiation into morphological forms that are best adapted to environmental adversities and other bacterial physiological events (Swift et al., 2001).

The QS system has been extensively studied in pathogenic and spoilage bacteria with the aim of elucidating the mechanisms involved in the regulation of infectious processes and in food spoilage (Jamuna Bai and Ravishankar Rai, 2011). Among the Salmonella genus, the QS systems have been examined in Salmonella Typhimurium, although different serovars of Salmonella spp presumably also exhibit the same three QS systems previously identified in this serovar (Halatsi et al., 2006).

The first QS system is incomplete in Salmonella spp because it fails to encode the AI-1 synthase homolog, termed LuxI, and expresses only the response regulator protein (SdiA), which is homologous to LuxR (Ahmer et al., 1998). Salmonella Typhimurium SdiA activity has been detected in the intestine of a turtle colonized by Aeromonas hydrophila (Smith 
et al., 2008) and in mice colonized by Yersinia enterocolitica (Dzsel et al., 2010). Both of these bacteria are pathogenic and produce AIs. In these competitive situations, Salmonella Typhimurium found in these animals' intestines, producing the QS response regulator protein, intercept and decode the AI-mediated messages from their competitors and develop strategies to survive and win the competition (Ahmer and Gunn, 2011; Kalia, 2013).

The second QS system identified in Salmonella is mediated by AI-2, whose internalization is dependent on an ATP-binding cassette $(\mathrm{ABC})$ transporter designated the Lsr transporter. The $l s r$ operon contains seven genes, $l s r A C D B F G E$, and its transcription is induced by AI-2 (Taga et al., 2001).

The third QS system found in Salmonella spp is mediated by AI-3, but little is known about this system. This communication mechanism exhibits characteristics that are shared with the AI-2 system, but unlike AI-2, the AI-3-mediated QS system also uses human hormones, epinephrine and norepinephrine, as signals (Walter and Sperandio, 2006).

The characterization of QS has revealed different levels of conservation in the three communication systems found among Gram-negative bacteria. According to Lerat and Moran (2004), the genes encoding the AI-1-, AI-2-, and AI-3-mediated QS systems are not fully conserved and can show variation within the same species. This fact suggests that losses or gains of genes have occurred, presumably through horizontal gene transfer (Lerat and Moran, 2004). However, recent studies have indicated that there is conservation of the gene sequences involved in QS within genera of bacteria and even between bacteria belonging to the same family (Gray and Garey, 2001).

Determining the molecular-level relationships between species is essential for making numerous biological inferences, and a proportionately large effort has been made to reconstruct this type of relationship among study organisms (Mcquiston et al., 2008). In this context, the aim of the current study was to characterize the AI-1- and AI-2-mediated QS systems of Salmonella Enteritidis PT4 578. A phylogenetic analysis of the nucleotide sequence encoding the response regulator protein of the AI-1-mediated QS mechanism (SdiA protein) was performed in an attempt to identify relationships between genera, species, and/or different serovars. Furthermore, a comparative analysis of the deduced amino acid sequence of the SdiA protein was performed with the aim of revealing how conserved these protein domains are.

\section{MATERIAL AND METHODS}

\section{Bacteria and growth conditions}

S. enterica serovar Enteritidis PT4 578 was isolated from chicken breast tissue and provided by FIOCRUZ (Fundação Oswaldo Cruz, Rio de Janeiro, Brazil). The other strains employed were Hafnia alvei and A. hydrophila 069, which were used as positive controls for acyl homoserine lactone production, and Chromobacterium violaceum CV026, as reporter bacterium for AI-1 (AHL)-mediated QS. Stocks of bacterial cultures were prepared in Luria Bertani (LB) broth medium ( $1 \%$ tryptone, $0.5 \%$ yeast extract, and $0.4 \% \mathrm{NaCl}), \mathrm{pH} 7.4$, containing a $33 \%$ glycerol $50(\mathrm{w} / \mathrm{v})$ solution in sterile water, and frozen at $-80^{\circ} \mathrm{C}$. Prior to each experiment, the cells were reactivated in LB or LB supplemented with specific antibiotic media, when needed, via incubation for $18 \mathrm{~h}$ at $37^{\circ} \mathrm{C}$ for Salmonella, or $30^{\circ} \mathrm{C}$ for $\mathrm{A}$. hydrophila and C. violaceum. 


\section{Screening for AHL production}

The Salmonella Enteritidis PT4 578 strain was incubated in $200 \mathrm{~mL} \mathrm{LB}$ medium at $37^{\circ} \mathrm{C}$ for $24 \mathrm{~h}$, followed by centrifugation at $10,000 \mathrm{~g}$ for $10 \mathrm{~min}$ in a Sorvall microcentrifuge (model MC12V; Dupont, USA). Extraction of AHL from the supernatant was performed according to the method of Ravn et al. (2001), with some modifications. The supernatant was poured into a separation funnel, supplemented with $200 \mathrm{~mL}$ ethyl acetate acidified with $0.5 \%$ formic acid, homogenized, and then left to stand for phase separation. The last two procedures were repeated three times. The organic phase was removed and added back to the separation funnel, together with an additional $200 \mathrm{~mL}$ ethyl acetate, and the stirring and standing procedures were repeated. The phase containing ethyl acetate was removed and transferred to a sterilized Erlenmeyer flask for further concentration in a rotary evaporator (model Q344B; Quimis, Brazil) at $42^{\circ} \mathrm{C}$, followed by resuspension in $600 \mu \mathrm{L}$ ethyl acetate and freezing at $-20^{\circ} \mathrm{C}$. The $A$. hydrophila 069 strain was used as a positive control for AHL production, and the procedure for the extraction of AHL from the culture supernatant was performed as described above. After the signaling molecules were obtained, they were identified by thin-layer chromatography (TLC).

The following AHLs were used as standards in the TLC analysis: N-hexanoyl homoserine lactone and N-butanoyl-DL-homoserine lactone (Fluka, Switzerland). The standards and samples were applied to reverse-phase TLC plates ( $\mathrm{C}_{18}$, RP 254s, Merck, Germany), measuring $20 \times 20 \mathrm{~cm}$, in volumes ranging from 10 to $20 \mu \mathrm{L}$. The chromatograms were developed in a methanol/water $(60: 40, \mathrm{v} / \mathrm{v})$ solvent system. At the end of the run time, the solvent was evaporated and the dry plates were coated with a bacterial culture prepared as follows: $20 \mathrm{~mL}$ C. violaceum 026 culture that had been activated for $24 \mathrm{~h}$ was used to inoculate $200 \mathrm{~mL} 0.65 \%$ LB agar. Following homogenization, the culture was evenly spread over the plate surface and enclosed with a safeguard to prevent agar spillage. Upon solidification of the medium, the plates were incubated at $37^{\circ} \mathrm{C}$ for 16 to $24 \mathrm{~h}$ in a properly closed humidified plastic container (Ravn et al., 2001; Christensen et al., 2003). In this assay, the production of violacein by $C$. violaceum resulted in violet spots on the plates, which is considered a positive result for the presence of AHLs (Shaw et al., 1997).

\section{Effect of AHL on Salmonella Enteritidis PT4 growth}

The effect of synthetic AHLs on Salmonella Enteritidis PT4 578 growth was assessed via a microplate assay using LB medium. Salmonella Enteritidis PT4 578 was grown for approximately $18 \mathrm{~h}$ at $37^{\circ} \mathrm{C}$ with stirring in $10 \mathrm{~mL}$ LB medium. The cells were collected by centrifugation in a Sorvall microcentrifuge (model MC12V, Dupont) at $4300 \mathrm{~g}$ for $10 \mathrm{~min}$. The supernatant was discarded, and the pellet was resuspended in $1.5 \mathrm{~mL} 0.85 \%$ saline solution and then centrifuged again at the same speed for the same time. Following this step, the supernatant was discarded and the absorbance of cells was standardized to prepare a population equivalent to $10^{7}$ colony-forming units $/ \mathrm{mL}$. A $20-\mu \mathrm{L}$ volume of this suspension was added to $180 \mu \mathrm{L} \mathrm{LB}$ medium distributed on a 96-well polystyrene microplate and supplemented with $100 \mathrm{nM}$ concentrations of the following AHLs: N-hexanoyl homoserine lactone (Fluka), N-octanoyl homoserine lactone (Fluka), N-decanoyl homoserine lactone (Sigma), and N-dodecanoyl homoserine lactone (Fluka). The microplate was subsequently incubated at $37^{\circ} \mathrm{C}$ without stirring and the absorbance was measured at $630 \mathrm{~nm}$ in a VERSA MAX enzyme- 
linked immunosorbent assay (ELISA) reader (Molecular Devices; Sunnyvale, CA, USA) every 30 min until the stationary phase was reached. Eight replicates and three repetitions of this experiment were conducted.

\section{Touchdown polymerase chain reaction (PCR) for amplification of AI-1- and AI- 2-mediated QS genes}

Characterization of the QS system was performed in Salmonella Enteritidis PT4 578 based on data from Salmonella Typhimurium. Genomic DNA extraction from Salmonella Enteritidis PT4 578 was performed using the Wizard Genomic DNA Purification kit (Promega, USA). Four Touchdown PCR programs were created according to Korbie and Mattick (2008) to amplify the 11 genes involved in the AI-1 and AI-2-mediated QS mechanisms in Salmonella Enteritidis PT4 578, which were initially identified in Salmonella Typhimurium (Taga et al., 2001), corresponding to the operon $l s r$ genes (10 genes) and sdiA. Pairs of oligonucleotides (Table 1) were designed based on the Salmonella Enteritidis PT4 P125109 genome deposited in GenBank (accession No. AM 933172.1) by Thomson et al. (2008) and Salmonella Typhimurium LT2 sequences (accession No. AE 006468.1).

The Touchdown PCR program is based on three phases. The first phase consisted of a denaturation step at $95^{\circ} \mathrm{C}(3 \mathrm{~min})$, followed by 10 cycles of another denaturation step at $95^{\circ} \mathrm{C}(30 \mathrm{~s})$, an annealing step with an increase of $10^{\circ} \mathrm{C}$ from the desired annealing temperature $(\mathrm{Tm})$, and a final extension step at $72^{\circ} \mathrm{C}(70 \mathrm{~s})$. The second phase comprised a denaturation step at $95^{\circ} \mathrm{C}(30 \mathrm{~s})$, followed by a sequence of annealing cycles for $40 \mathrm{~s}$ each starting at the desired Tm plus $5^{\circ} \mathrm{C}$ and decreasing by $1{ }^{\circ} \mathrm{C}$ each cycle for six consecutive cycles until the desired Tm was reached, with an extension cycle at $72^{\circ} \mathrm{C}(70 \mathrm{~s})$; the entire sequence was repeated 19 times when the desired Tm was reached. The third phase consisted of one cycle at $72^{\circ} \mathrm{C}(5 \mathrm{~min})$. The programs differed according to the desired $\mathrm{Tm}$ of $48,50,56$, or $59^{\circ} \mathrm{C}$. The PCR amplifications were performed in a $25-\mu \mathrm{L}$ volume with $100 \mathrm{ng}$ DNA, $1 \mu \mathrm{L}$ each oligonucleotide, $10 \mathrm{pmol}$ forward and reverse primer, 12.5 $\mu \mathrm{L}$ GoTaq Colorless Master Mix (Promega), and $9.5 \mu \mathrm{L} \mathrm{H}_{2} \mathrm{O}$. The obtained PCR products were purified using the Wizard SV Gel and PCR Clean-up System (Promega) and sent for sequencing at Macrogen Inc. (Seoul, South Korea).

\section{In silico analysis}

\section{AI-2-mediated QS model}

The targeted genes were amplified using the Touchdown PCR technique (described above) and sequenced to assess whether the organization of the genes belonging to the AI2-mediated QS system of Salmonella Enteritidis PT4 578 corresponded to those of Salmonella Typhimurium reported by Taga et al. (2001). These sequences were identified in the Salmonella Enteritidis PT4 P125109 (AM933172.1) genome deposited by Thomson et al. (2008) using the BLASTn tool (Altschul et al., 1990). The positions and directions of the genes were identified using the National Center for Biotechnology Information (NCBI) Graphics tool for sequenced genomes. 


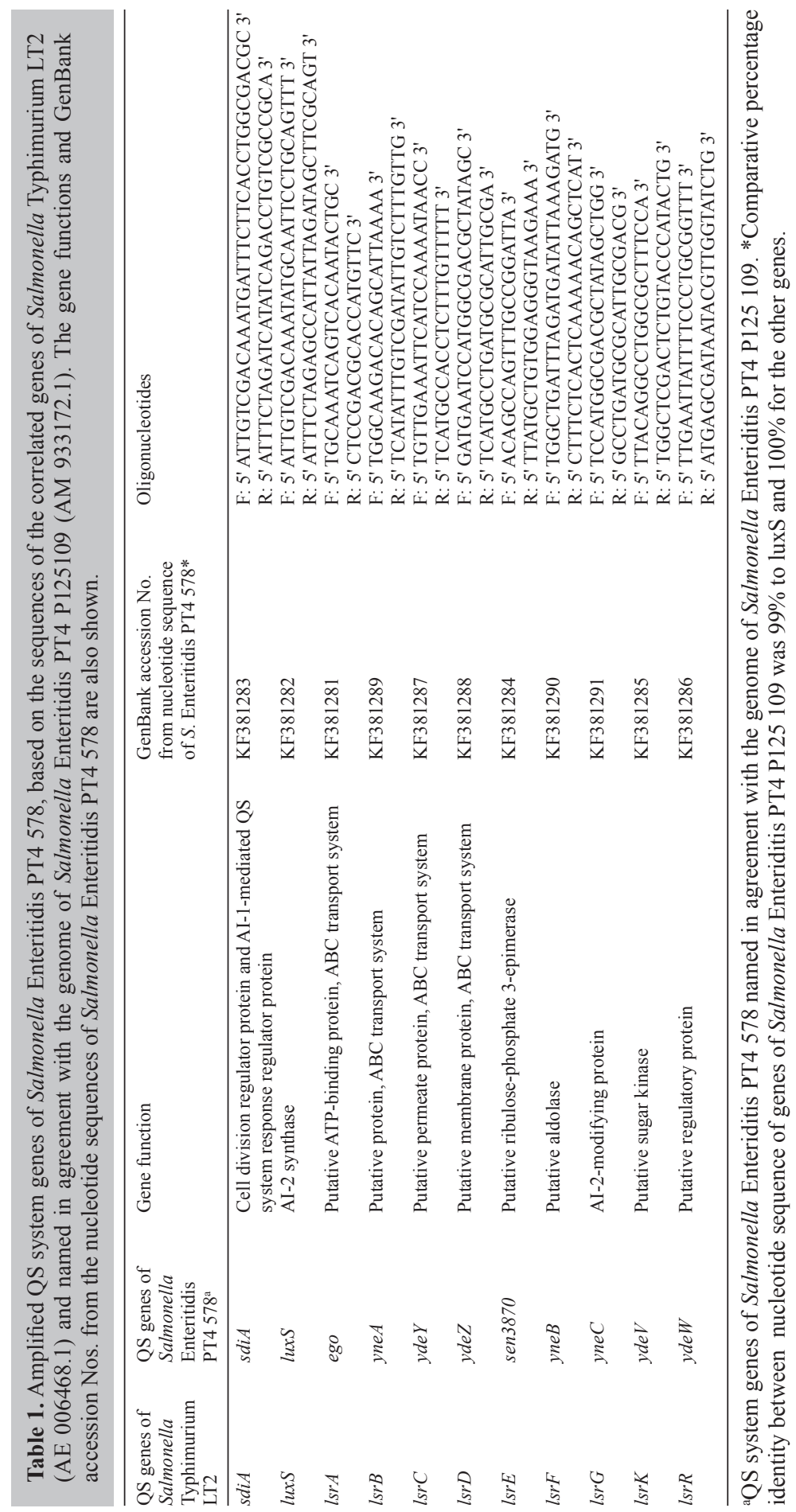




\section{Phylogenetic analysis of the sdiA gene and comparative analysis of the deduced amino acid sequence of the SdiA protein}

A phylogenetic analysis was performed with the aim of comparing the nucleotide sequences encoding the response regulator protein of the QS systems (SdiA). The sequences used in the analysis were obtained from the GenBank database at the NCBI web page, and the included bacterial species, followed by their accession Nos. (Table 2). The nucleotide sequence encoding the SdiA protein of the Salmonella Enteritidis PT4 578 study isolate was also included.

Table 2. Bacterial species and GenBank accession Nos. of nucleotide sequences used in phylogenetic analysis, encoding the SdiA protein, a response regulator of the QS systems.

\begin{tabular}{|c|c|}
\hline Bacterial species & GenBank (accession No.) SdiA \\
\hline Citrobacter freundii $4747 \mathrm{CFAA}$ & ZP_09336017.1 \\
\hline Citrobacter rodentium ICC168 & YP_003365526.1 \\
\hline Citrobacter youngae ATCC 29220 & ZP_06352425.1 \\
\hline Cronobacter turicensis Z3032 & YP_003210982.1 \\
\hline Escherichia coli BW2952 & YP_002926921.1 \\
\hline Escherichia coli DH1 & YP_006129257.1 \\
\hline Escherichia coli E101 & EH_O00167.1 \\
\hline Escherichia coli $\mathrm{O} 103 \mathrm{H} 2$ & YP_003222098.1 \\
\hline Escherichia coli O55 H7 CB9615 & YP_003499911.1 \\
\hline Escherichia coli TW14359 & YP_003078403.1 \\
\hline Enterobacter cloacae ATCC 13047 & YP_003611882.1 \\
\hline Enterobacter hormaechei ATCC 49162 & ZP-08498664.1 \\
\hline Erwinia billingiae Eb661 & YP_003740953.1 \\
\hline Klebsiella pneumoniae MGH 78578 & YP_001336067.1 \\
\hline Pantoea ananatis LMG 20103 & YP_003519711.1 \\
\hline Salmonella bongori NCTC 12419 & YP_004730634.1 \\
\hline Salmonella Choleraesuis SCSA50 & ZP_09760024 \\
\hline Salmonella Dublin SD3246 & ZP_09763785.1 \\
\hline Salmonella Dublin CT02021853 & YP 002215136 \\
\hline Salmonella Agona & YP_002146073 \\
\hline Salmonella Enteritidis P125109 & YP_002243187 \\
\hline Salmonella Enteritidis PT4 578 & $\mathrm{KF} 381283.1^{\wedge}$ \\
\hline Salmonella Gallinarum & YP 002226156 \\
\hline Salmonella Heidelberg B182 & AF_H45905.1 \\
\hline Salmonella Heidelberg SL476 & YP_002046001 \\
\hline Salmonella Newport SL254 & $\mathrm{AC}^{-} \mathrm{F} 63987.1$ \\
\hline Salmonella Paratyphi A & YP_150210 \\
\hline Salmonella Paratyphi C & YP 002637347 \\
\hline Salmonella Schwarzengrund & $\mathrm{ZP}-02664244$ \\
\hline Salmonella Schwarzengrundstr CVM19633 & YP_002114983 \\
\hline Salmonella Typhi & CA_D05699 \\
\hline Salmonella Typhi Ty2 & NP_804754 \\
\hline Salmonella Typhimurium LT2 & LT2 NP 460903 \\
\hline Salmonella Typhimurium SL1344 & SL1344 YP_005181773 \\
\hline Shigella boydii & YP_0018797705 \\
\hline Shigella sonnei & YP 310157 \\
\hline Yokenella regensburgei ATCC 43003 & ZP_09390474 \\
\hline
\end{tabular}

$\triangle$ Nucleotide sequence encoding the SdiA protein of the Salmonella Enteritidis PT4 578 study isolate.

Manual alignment of these nucleotide sequences was performed using the Mega 5.0 software (Kumar et al., 2008; Tamura et al., 2011) according to the deduced amino acid sequence corresponding to the SdiA protein. These sequences were pooled to perform phylogenetic inference through Bayesian inference (Yang and Rannala, 1997).

Bayesian inference was performed using the MrBayes 3.1 software (Huelsenbeck and Ronquist, 2001), and the evolutionary model SYM $+\mathrm{I}+\mathrm{G}$ was selected using the MrMod- 
eltest v2 software (Nylander, 2004). Two independent runs with four Monte Carlo Markov chains were performed for 10 million generations, and the trees were sampled and retained at every 1000th generation. The first 1 million tree samples were discarded in the burn-in phase, and the remaining trees were summarized to generate a consensus of the majority tree.

The alignment and a comparative analysis of the deduced amino acid sequence of the SdiA protein from 12 isolates and the study isolate Salmonella Enteritidis PT4 578 were performed using the ClustalW2 software (Larkin et al., 2007). The 12 isolates, obtained from GenBank were as follows: Citrobacter youngae ATCC 29220 (ZP 06352425.1); Citrobacter turicensis Z3032 (YP 003210982.1); Escherichia coli O55H7 CB̄9615 (YP 003499911.1); Enterobacter cloacae ATCC 13047 (YP_003611882.1); Klebsiella pneumoniae MGH 78578 (YP_001336067.1); Pantoea ananatis LMG 20103 (YP_003519711.1); Salmonella bongori NCTC 12419 (YP_004730634.1); Salmonella Enteritidis P125109 (YP_002243187); Salmonella Typhi (CA_D05699); Salmonella Typhimurium LT2 (NP_460903); Shigella boydii (YP_001879705); and Yokenella regensburgei ATCC 43003 (ZP_09390474)]. Characterization of the protein functional domains was performed using the Conserved Domain Database tool (Marchler-Bauer et al., 2011).

\section{RESULTS AND DISCUSSION}

\section{Quorum sensing in Salmonella Enteritidis PT4 578}

No gene homologous to luxI was found in the Salmonella Enteritidis PT4 578 genome using the BLASTn tool. PCR was performed with pairs of oligonucleotides targeting the luxI homologs of the H. alvei, E. cloacae, and Salmonella Enteritidis PT4 578 enterobacteria, and no resultant DNA fragment was detected on $1.5 \%$ agarose gels. The same procedure was performed with $A$. hydrophila 069 , in which a luxI gene fragment was amplified. These results indicate that Salmonella Enteritidis PT4 578 lacks AI-1 synthase. Confirmation that this serovar failures to produce the signal molecule (AI-1) was performed through TLC, as no spot relative to induction of violacein production by $C$. violaceum was detected (Figure 1).

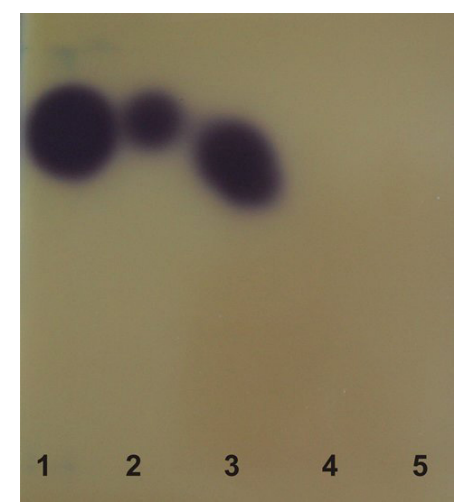

Figure 1. Thin-layer chromatography (TLC) of AHL extracts prepared from Salmonella Enteritidis PT4 578 and Aeromonas hydrophila 069 grown in Luria Bertani medium for $18 \mathrm{~h}$, revealed using the reporter strain Chromobacterium violaceum CV026. Lane $1=1 \mathrm{mg} / \mathrm{mL}$ N-butanoyl homoserine lactone standard $(6 \mu \mathrm{L})$; lane $2=0.1 \mathrm{mg} / \mathrm{mL} \mathrm{N}$-hexanoyl homoserine lactone standard $(6 \mu \mathrm{L})$; lane $3=$ A. hydrophila 069 cell-free supernatant extract $(60 \mu \mathrm{L})$; lane $4=$ Culture medium extract $(60 \mu \mathrm{L})$; and lane $5=$ Salmonella Enteritidis PT4 578 cell-free supernatant extract $(60 \mu \mathrm{L})$. 
An incomplete AI-1-mediated QS system was first described in E. coli, which lacks LuxI, an AI-1 synthase but has the LuxR homolog, termed SdiA (Sitnikov et al., 1996). The presence of sdiA in the Salmonella Typhimurium genome was identified through alignment analyses performed by Ahmer et al. (1998). The lack of a luxI gene homolog in the Salmonella Enteritidis PT4 578 genome and confirmation that this serovar fails to produce AHL do not preclude it from responding to AHLs produced by other microorganisms.

Growth curves for this pathogen in the presence of different AHLs were designed with the aim of assessing whether the AHLs affect Salmonella Enteritidis PT4 578 growth. In these assays, a $100 \mathrm{nM}$ concentration of the signaling molecules did not noticeably affect bacterial growth (Figure 2).

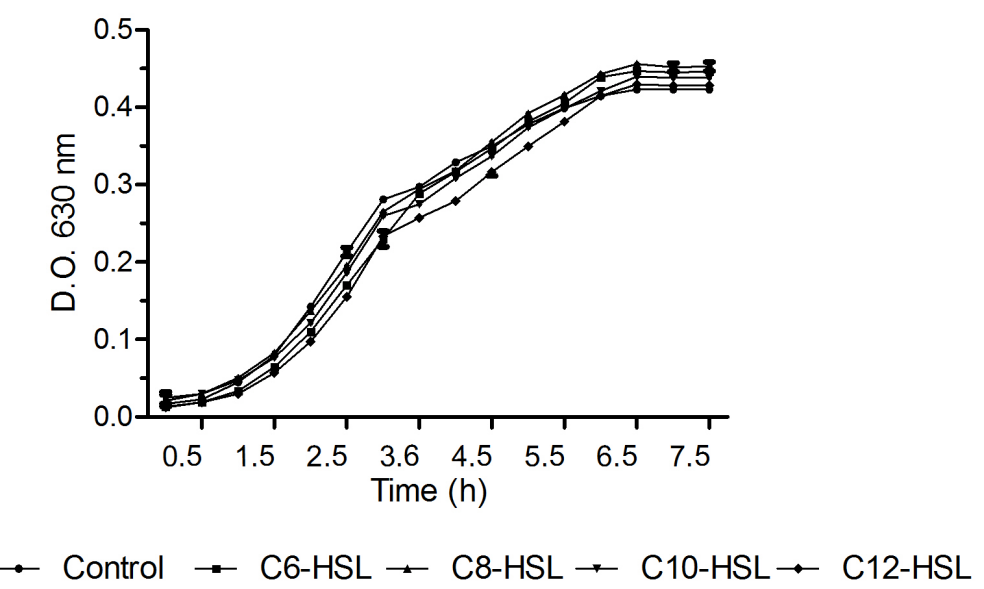

Figure 2. Salmonella Enteritidis PT4 578 growth over $7.5 \mathrm{~h}$ in Luria Bertani medium (control) and Luria Bertani media with different AHLs at a concentration of $100 \mathrm{nM}$. AHLs: C6-N-hexanoyl homoserine lactone, C8-N-octanoyl homoserine lactone, $\mathrm{C} 10-\mathrm{N}$-decanoyl homoserine lactone, and $\mathrm{C} 12-\mathrm{N}-$ dodecanoyl homoserine lactone.

Michael et al. (2001) showed that, depending on the AHL assessed, a concentration of $1 \mathrm{nM}$ suffices to induce sdiA gene expression in Salmonella Typhimurium. Confirmation that a $100 \mathrm{nM}$ concentration of different AHLs does not negatively affect the microorganism's growth is essential for studying phenotypes regulated by these AIs because it enables the detection of phenotypes stimulated by this signal.

Genes encoding products belonging to AI-1- and AI-2-mediated QS systems were identified in the Salmonella Typhimurium LT2 (AE 006468.1) genome, and the corresponding genes were recognized in Salmonella Enteritidis PT4 P125109 (AM 933172.1) using the BLASTN tool (Table 1). The same genes were found in Salmonella Enteritidis PT4 578 after sequencing (Table 1). All sequenced gene products from Salmonella Enteritidis PT4 578 were deposited and showed a greater than $99 \%$ identity to gene sequences of Salmonella Enteritidis PT4 P125109 (AM 933172.1).

The sdiA gene, encoding the response regulator protein SdiA, is present in the Salmonella Enteritidis PT4 P125109 (AM 933172.1) genome sequenced by Thomson et al. (2008) and this gene was also identified in Salmonella Enteritidis PT4 578, containing 723 bp (Table 1).

The SdiA protein is a sensor of AHLs produced by other bacterial species, possibly in mammalian gastrointestinal tracts, as described by Ahmer and Gunn (2011). SdiA positively 
regulates two loci in Salmonella Typhimurium, the $r c k$ operon and the $\operatorname{srg} A B C$ operon, both of which were found in a virulence plasmid. The first is responsible for increasing resistance to host defense responses, whereas the latter operon lacks a given function (Ahmer et al., 1998; Michael et al., 2001).

Another gene potentially regulated by $\mathrm{SdiA}$ is $\operatorname{srg} E$, which was acquired by horizontal transfer and has an unknown function (Sabag-Daigle et al., 2012). However, according to in silico nucleotide sequence analyses performed by the same authors, the SrgE protein may be involved in type III secretion systems (Sabag-Daigle et al., 2012).

The other genes identified in the genome of Salmonella Enteritidis PT4 578 (Table 1) belong to the AI-2-mediated system, in which luxS encodes AI-2 synthase, and the other genes belong to an operon responsible for transporting AI-2. An in silico analysis based on the identification and position of the AI-2-mediated QS system genes was performed to predict the model of genome organization in Salmonella Enteritidis PT4 578 from the genome of Salmonella Enteritidis P125109 (AM 933172.1). These genes are found in the region extending from 4.1485 to $4.1585 \mathrm{Mbp}$, and these data were subjected to comparison with the model of the AI-2-mediated QS system in Salmonella Typhimurium proposed by Taga et al. (2001).

Following a detailed analysis, the organization of the AI-2-mediated QS system was concluded to match in the two serovars. In addition, the model suggested for Salmonella Enteritidis PT4 578 matched the model proposed for the organization of the $l s r$ operon in Salmonella Typhimurium by Taga et al. (2001) and these results show that the AI-2-mediated mechanism is conserved between these serovars (Figure 3). The suggested model (Figure 3) will enable the investigation of whether the AI-2-regulated phenotypes observed in Salmonella Typhimurium exhibit the same mode of regulation in Salmonella Enteritidis PT4 578.

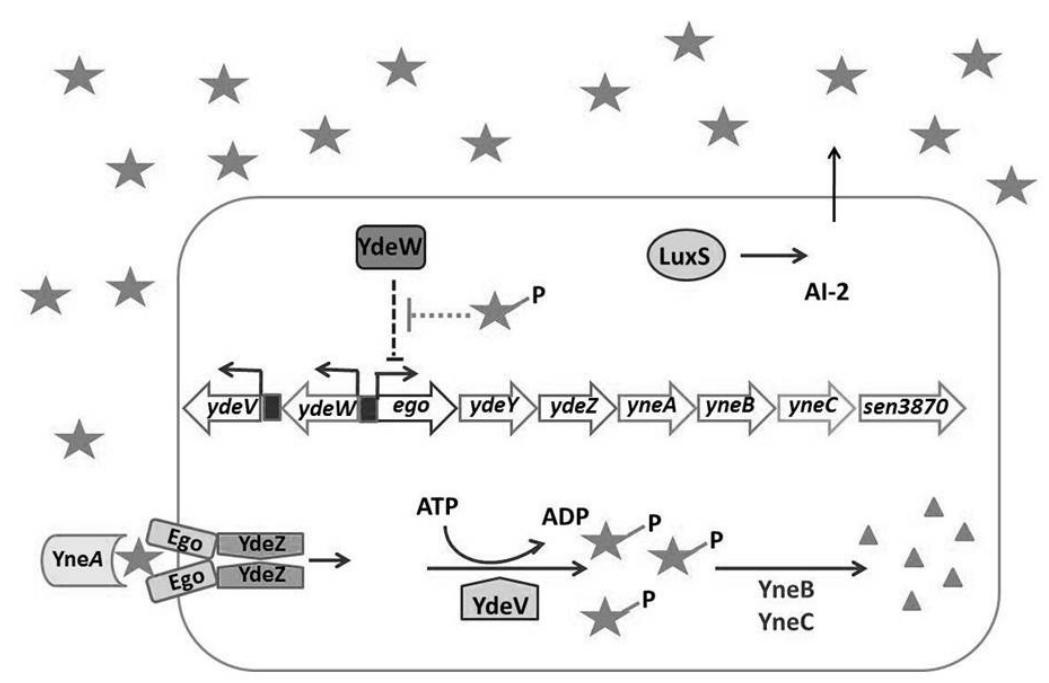

Figure 3. Suggested model for the regulation of the AI-2-mediated QS system in Salmonella Enteritidis PT4 578. $\mathrm{AI}-2$ (star) is synthesized by LuxS and accumulates in the extracellular medium. The basal expression of the ABC transport system (YneA, Ego, and YdeZ), encoded by the operon found in this system, enables some AI-2 to enter the cytoplasm, wherein it is phosphorylated by $\mathrm{YdeV}$. The phosphorylated AI-2 induces operon transcription through YdeW inactivation. At high concentrations, phosphorylated AI-2 is processed by YneB and YneC (modified model of Taga et al., 2001). The copyrights of the model proposed by Taga et al. (2001) were kindly provided for publication in this study. 
The alignment of Salmonella Enteritidis PT4 578 genes performed with the genome of the P125109 serotype enabled us to identify AI-2-mediated QS genes using the BLASTn tool and to confirm the presence of only one copy of the genes in the genome of Salmonella Enteritidis PT4 578. In this serovar, the lsr operon is represented by the $y d e V, y d e W$, ego, $y d e Y$, ydeZ, yneA, yneB, yneCv, and sen 3870 genes (Figure 3), which are responsible for encoding the AI-2 transport and modulation proteins. The luxS gene encoding the AI-2 synthase is also present in addition to these genes, although situated in another portion of the genome.

In the AI-2-mediated QS system, this signaling molecule is synthesized by LuxS and released into the extracellular medium. High concentrations of this molecule in the external environment and basal expression of the genes encoding the ABC transport system enable AI-2 entry into the cytoplasm. The transport system is formed by YneA, a homolog of the LsrB protein; Ego, homologous to LsrA; and YdeZ, homologous to LsrD, from the lsr operon of Salmonella Typhimurium (Taga et al., 2001). The YdeV protein, corresponding to the LsrK protein of Salmonella Typhimurium, is responsible for phosphorylating AI-2, and this molecule can therefore act as a transcriptional regulator, inducing the transcription of this operon by inactivating YdeW, corresponding to LsrR. The AI-2 concentration is decreased in the presence of YneB and YneC, which are homologous to the LsrF and LsrG proteins, respectively, through unknown mechanisms. No product from the other genes belonging to the lsr operon of Salmonella Typhimurium (Taga et al., 2001) was directly correlated with the AI-2-mediated system of Salmonella Enteritidis PT4 578. However, possible functions of these genes are highlighted in Table 1.

$\mathrm{AI}-2$ is regulated by the $I S A C D B F G E$ operon in the model proposed for Salmonella Typhimurium (Taga et al., 2001). The first four genes, $l s r A C D B$, encode the AI-2 import components. The function of the other three $\operatorname{srFGE}$ genes is unknown, although nucleotide sequence analyses suggest that $l s r F$ encodes an aldolase, while $l s r G$ encodes a conserved protein of unknown function, and $l s r E$ is homologous to rpe, encoding a ribulose-phosphate isomerase (Taga et al., 2001). The $l_{s r} R$ gene, located upstream of $l_{s r} A$, encodes a transcriptional repressor and is transcribed differently than the $l s r$ operon. LsrR represses $l s r$ operon transcription in the absence of AI-2, and its presence results in derepression (Taga et al., 2001).

\section{Phylogenetic analysis of the sdiA gene and comparative analysis of the deduced amino acid sequence of the SdiA protein}

A phylogenetic analysis was performed to obtain evidence of how conserved the nucleotide sequences of the gene encoding the response regulator protein SdiA (homologous to LuxR) are and to predict relationships between the bacterial genera, species, and serovars available in the database.

The phylogenetic relationships of the nucleotide sequence of the SdiA protein are shown in the majority consensus tree generated via Bayesian inference (Figure 4). The values next to each ancestor node, ordered from left to right, correspond to the $a$ posteriori probability value. The branches of trees whose a posteriori probability values were below 0.95 were omitted because this indicated a low reliability of the branch, as supported by inference. 


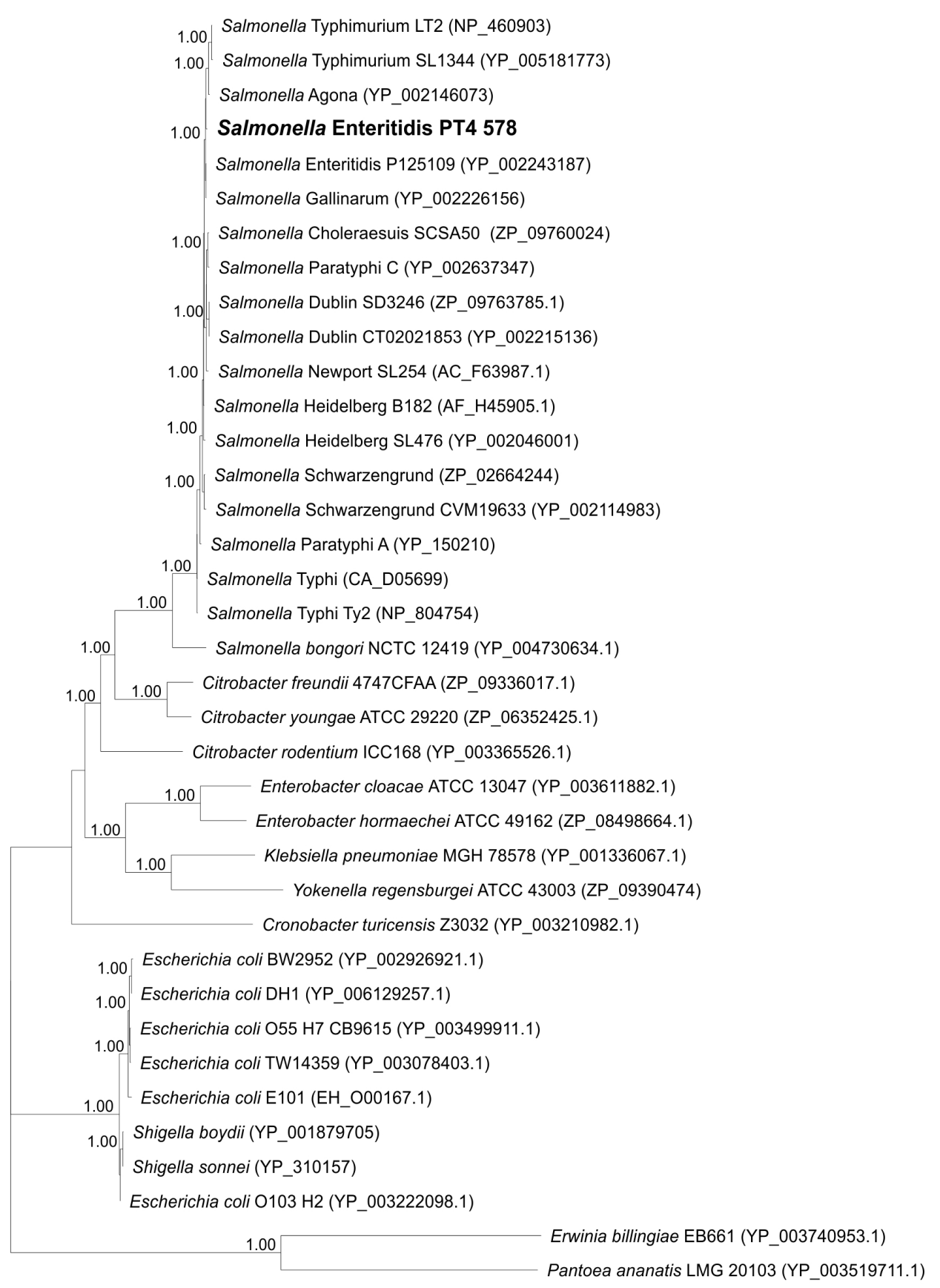

Figure 4. Phylogenetic tree constructed via Bayesian inference using the nucleotide sequences of the genes encoding the SdiA protein in 36 bacterial isolates found in GenBank (accession No. in parentheses) and the study isolate Salmonella Enteritidis PT4 578 (in bold). The a posteriori probability value obtained using Bayesian inference is shown, from left to right, beside each ancestor node. Values below 0.95 were omitted from the tree. The bar indicates a substitution estimated every $10 \mathrm{bp}$. 
The constructed tree (Figure 4) shows that the nucleotide sequence of genes encoding the response regulator protein SdiA are conserved among organisms of the same genus (Enterobacter, Escherichia, Salmonella, and Shigella) based on the a posteriori probability value. An exception should be made for E. coli O103: H2, which is grouped with representatives of the genus Shigella. The Pantoea and Erwinia genera are phylogenetically so distant that they were inevitably used as an outgroup to highlight the differences among the other genera.

Similar to other QS proteins, the SdiA protein contains approximately 228 amino acids and consists of two domains: an N-terminal AI-binding domain (Figure 5) and a C-terminal domain (Figure 5), containing a helix-turn-helix motif involved in DNA binding (Nasser and Reverchon, 2007). AI binding in these proteins modulates the binding of the SdiA C-terminal protein domain to DNA. For example, in the case of the LuxR protein, an AHL binds to its N-terminal domain, leading to dimerization (Choi and Greenberg, 1991).

The SdiA protein N-terminal AI-binding domain is highly conserved within each bacterial genus (the Salmonella genus in this case, highlighted in white text in Figure 5). This indicates that each bacterial genus possibly responds to the same AIs. In the same way, the $\mathrm{N}$-terminal binding domains for the AIs vary greatly among different bacterial genera, possibly indicating that each genus has an inherent ability to respond to different types of AIs. However, this does not mean that different bacterial genera can respond to the same AI.

The SdiA protein DNA-binding domain is quite conserved in all of the bacteria evaluated (Figure 5). This is shown by the high frequency of the asterisks and colons, which indicate that the amino acid residues in the column were identical in all of the aligned sequences and that there were substitutions, albeit conserved, of residues, respectively.

A plausible explanation for the conservation of this gene and its product in environments characterized by high selective pressure imposed by competition between bacterial species and/or genera is the ability of the SdiA protein to detect and respond to AHL signal molecules produced by different bacteria (Case et al., 2008). This ability confers advantages on the bacteria because it enables them to regulate their increase in virulence, as in the wellstudied case of Salmonella Typhimurium and in biofilm formation by E. coli, both of which are crucial phenotypes to overcome competition and infect the host (Case et al., 2008).

QS response regulators similar to LuxR act simultaneously as sensors and transcriptional factors (Choi and Greenberg, 1991). The AI- and DNA-binding domains are fused in this signal transduction system. For this reason, it is referred to as a one-component signal transduction system, which has been shown to be the most common type of signal transduction system (Madan Babu and Teichmann, 2003).

A phylogenetic analysis performed by Case et al. (2008) showed that 45 bacteria of all of the bacterial genomes deposited in the database until 2008 included a LuxR homolog, albeit without the corresponding AHL synthase.

Although it is unknown whether all LuxR homologs are actually functional, this profile may be prevalent among proteobacteria, and $26 \%$ of all of the sequenced bacterial genomes from this group exhibit the complete QS system, including genes encoding the AI synthase and the response regulator protein, while $17 \%$ present an incomplete QS, failing to synthesize the signaling molecule but showing the potential to spy on the AHL-mediated QS system of other proteobacteria (Case et al., 2008). Some of the species chosen for the phylogenetic analysis performed here exhibited the complete AI-1-mediated QS system; that is, they presented both the synthase-encoding gene (luxI) and the response regulator protein-encoding gene $($ luxR). The nucleotide sequence of the LuxR protein, which belongs to the complete sys- 
tem, despite being considered homologous to SdiA (the regulatory protein of the incomplete system), shows a low identity to SdiA (data not shown), which can be explained by the greater flexibility of the SdiA protein in detecting and responding to signal molecules produced by other bacterial species and/or genera (Gray and Garey, 2001).

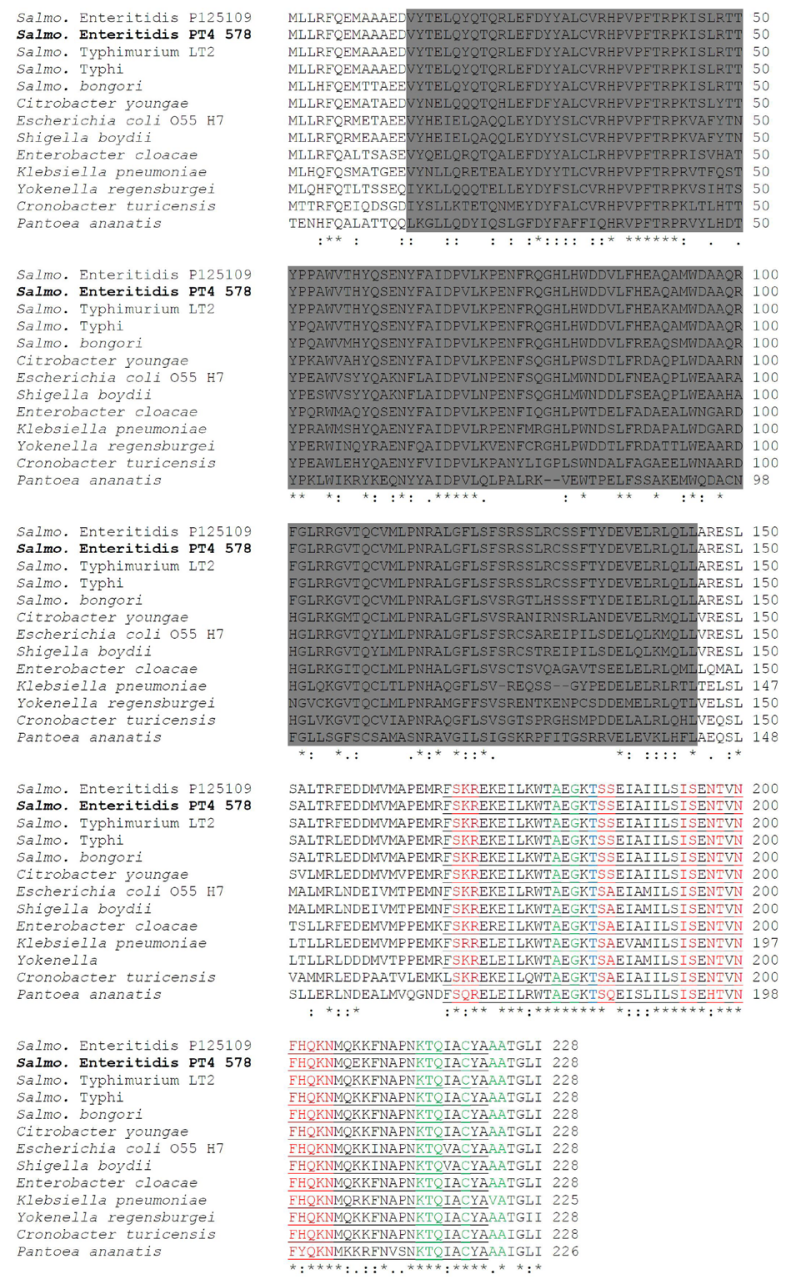

Figure 5. Deduced amino acid sequence alignment of the SdiA protein of 12 isolates from GenBank (for accession No., refer to the Material and Methods) and the study isolate in Salmonella Enteritidis PT4 578 (in bold) using ClustalW2 software. The two main domains of the SdiA protein were elucidated using the Conserved Domain Database tool. The autoinducer-binding N-terminal domain is shaded in dark gray, and the DNA-binding C-terminal domain, showing a helix-turn-helix motif, is shaded in light gray. The amino acid residues within the autoinducerbinding domain that were identical between species and serovar representatives of the genus Salmonella are highlighted in white. The DNA-binding amino acid residues within the DNA-binding domain are shown in red, while the residues involved in the dimerization interface are presented in green, and the residues involved both in DNA-binding and dimerization are indicated in blue. Symbols: asterisk means that the amino acid residues in the column were identical in all sequences aligned; colon means there were substitutions of residues, but the substitutions were conserved; and dot indicates semi-conserved substitutions. 
The genetic exchange capacity of bacteria can be demonstrated by the QS mechanism they exhibit. The presence of two components (luxI, encoding an AI-1 synthase protein, and luxR, encoding a response regulator protein that acts as a transcriptional factor, activating or repressing gene expression) is observed when the AI-1-mediated QS mechanism is analyzed, as originally described in V. fischeri by Nealson and Hastings (1979). However, this system is incomplete in some microorganisms, and it may have arisen through horizontal gene transfer (Gray and Garey, 2001).

Bacteria of the Citrobacter genus are enteric pathogens that show a similar mode of action to enteropathogenic E. coli and exhibit the complete QS system, consisting of croI (luxI) and croR (luxR) (Coulthurst et al., 2007). Erwinia spp also belong to the family Enterobacteriaceae, including species with a verified complete system, such as Erwinia carotovora, showing a QS mechanism that involves both carI (luxI) and carR (luxR). Only the gene encoding the SdiA-homolog regulator protein was detected in Erwinia amylovora (Smith et al., 2008), whereas the complete system consisting of esaI, esaR, eagI, and eagR was found in the genus Pantoea (Gray and Garey, 2001). However, there is little information available in the literature about the other genera analyzed, including Cronobacter spp (formerly Enterobacter sakazakii), Klebsiella, and Yokenella, in which the complete and incomplete QS systems are still poorly understood (Araújo et al., 2012).

In conclusion, Salmonella Enteritidis PT4 578 exhibits AI-1- and AI-2-mediated QS systems. To our knowledge, these systems were characterized for the first time in Salmonella Enteritidis in the present study, and they are similar to the QS systems described for Salmonella Typhimurium.

The nucleotide sequences encoding the SdiA protein of Salmonella Enteritidis PT4 578 are grouped with the other serovars of $S$. enterica, including Typhimurium. Thus, previously elucidated phenotypes known to be regulated by AI-1-mediated QS in Salmonella Typhimurium may be extended to studies in Salmonella Enteritidis.

Data obtained from the phylogenetic analysis of the nucleotide sequence of the SdiA protein and from a comparative alignment of its deduced amino acid sequence clearly show that both the gene and the protein, including its domains, are conserved within a bacterial genus. Thus, bacteria from the same genus can respond to the same AIs. However, this does not preclude the possibility that different bacterial genera may respond to the same AI, which may confer a competitive advantage on these bacteria, given their ability to respond to the signal released by the quorum comprised of other organisms.

The characterization of the QS mechanisms of the human pathogen Salmonella Enteritidis performed in the current study will enable future clarification of new control strategies in potential AI-1- or AI-2-regulated phenotypes. Moreover, the combination of conventional antibiotics and the development of quorum inhibitors may result in greater control of these pathogenic agents by preventing biofilm formation, overcoming resistance to antibiotics, or even reducing the microorganism virulence.

\section{ACKNOWLEDGMENTS}

Research supported by the following Brazilian agencies: Minas Gerais Science Foundation (FAPEMIG - Fundação de Amparo à Pesquisa do Estado de Minas Gerais); Brazilian Federal Agency of Support and Evaluation of Postgraduate Education (CAPES - Coordenação de Aperfeiçoamento de Pessoal de Nível Superior); and National Council of Scientific and 
Technological Development (CNPq - Conselho Nacional de Desenvolvimento Científico e Tecnológico). We thank John Wiley \& Sons Ltd (www.wiley.com) for permission to publish the model of quorum sensing of Salmonella Enteritidis (Figure 3) based on the model of Salmonella Typhimurium, previously published in Molecular Microbiology by Taga et al. (2001) [Article: The LuxS-dependent autoinducer AI-2 controls the expression of an ABC transporter that functions in AI-2 uptake in Salmonella Typhimurium]. Please consult John Wiley \& Sons for copyright information regarding Figure 3 in this study, and for permission to photocopy or otherwise reproduce this material, except for versions made by non-profit organizations for use by the blind, visually impaired, and other persons with print disabilities (VIPs).

\section{REFERENCES}

Ahmer BM and Gunn JS (2011). Interaction of Salmonella spp. with the intestinal microbiota. Front. Microbiol. 2: 101 Doi: $10.3389 /$ fmicb.2011.00101.

Ahmer BM, van Reeuwijk J, Timmers CD, Valentine PJ, et al. (1998). Salmonella Typhimurium encodes SdiA homolog, a putative quorum sensor of the LuxR family that regulates genes on the virulence plasmid. J. Bacteriol. 180: 1185-1193.

Altschul SF, Gish W, Miller W, Myers EW, et al. (1990). Basic local alignment search tool. J. Mol. Biol. 215: 403-410.

Araújo FD, Esper LM, Kuaye AY, Sircili MP, et al. (2012). N-Acyl-homoserine lactones from Enterobacter sakazakii (Cronobacter spp.) and their degradation by Bacillus cereus enzymes. J. Agric. Food Chem. 60: 585-592.

Case RJ, Labbate M and Kjelleberg S (2008). AHL-driven quorum-sensing circuits: their frequency and function among the Proteobacteria. ISME J. 2: 345-349.

Choi SH and Greenberg EP (1991). The C-terminal region of the Vibrio fischeri LuxR protein contains an inducerindependent lux gene activating domain. Proc. Natl. Acad. Sci. U. S. A. 88: 11115-11119.

Christensen AB, Riedel K, Eberl L, Flodgaard LR, et al. (2003). Quorum-sensing-directed protein expression in Serratia proteamaculans B5a. Microbiology 149: 471-483.

Coulthurst SJ, Clare S, Evans TJ, Foulds IJ, et al. (2007). Quorum sensing plays an unexpected role in virulence in the model pathogen, Citrobacter rodentium. EMBO Rep. 8: 698-703.

De Oliveira DC, Fernandes Júnior A, Kaneno R, Silva MG, et al. (2014). Ability of Salmonella spp. to produce biofilm is dependent on temperature and surface material. Foodborne Pathog. Dis. 11: 478-483.

Fuqua WC, Winans SC and Greenberg EP (1994). Quorum sensing in bacteria: the LuxR-LuxI family of cell densityresponsive transcriptional regulators. J. Bacteriol. 176: 269-275.

Gray KM and Garey JR (2001). The evolution of bacterial LuxI and LuxR quorum sensing regulators. Microbiol. 147: 2379-2387.

Halatsi K, Oikonomou I, Lambiri M, Mandilara G, et al. (2006). PCR detection of Salmonella spp. using primers targeting the quorum sensing gene sdiA. FEMS Microbiol. Lett. 259: 201-207.

Huelsenbeck JP and Ronquist F (2001). MRBAYES: Bayesian inference of phylogenetic trees. Bioinformatics. 17: 754-755.

Jamuna Bai A and Ravishankar Rai V (2011). Bacterial quorum sensing and food industry. Compr. Rev. Food Sci. Food Safety. 10: 184-194.

Kalia VC (2013). Quorum sensing inhibitors: An overview. Biotechnol. Adv. 31: 224-245.

Korbie DJ and Mattick JS (2008). Touchdown PCR for increased specificity and sensitivity in PCR amplification. Nat. Protoc. 3: 1452-1456.

Kumar S, Nei M, Dudley J and Tamura K (2008). MEGA: a biologist-centric software for evolutionary analysis of DNA and protein sequences. Brief Bioinform. 9: 299-306.

Larkin MA, Blackshields G, Brown NP, Chenna R, et al. (2007). Clustal W and Clustal X version 2.0. Bioinformatics 23: 2947-2948.

Lerat E and Moran NA (2004). The evolutionary history of quorum sensing in bacteria. Mol. Biol. Evol. 21: 903-913.

Maciel BM, Dias JC, Romano CC, Sriranganathan N, et al. (2011). Detection of Salmonella Enteritidis in asymptomatic carrier animals: comparison of quantitative real-time PCR and bacteriological culture methods. Genet. Mol. Res. 10: 2578-2588.

Madan Babu M and Teichmann SA (2003). Evolution of transcription factors and the gene regulatory network in Escherichia coli. Nucleic Acids Res. 31: 1234-1244.

March JC and Bentley WE (2004). Quorum sensing and bacterial cross-talk in biotechnology. Curr. Opin. Biotechnol. 15: 495-502. 
Marchler-Bauer A, Lu S, Anderson JB, Chitsaz F, et al. (2011). CDD: a Conserved Domain Database for the functional annotation of proteins. Nucleic Acids Res. 39 (Database issue): D225-229. doi: 10.1093/nar/gkq1189.

McQuiston JR, Herrera-Leon S, Wertheim BC, Doyle J, et al. (2008). Salmonella species and subspecies determined from four housekeeping genes and evidence of lateral gene transfer events. J. Bacteriol. 190: 7060-7067.

Michael B, Smith JN, Swift S, Heffron F, et al. (2001). SdiA of Salmonella enterica is a LuxR homolog that detects mixed microbial communities. J. Bacteriol. 183: 5733-5742.

Miller MB and Bassler BL (2001). Quorum sensing in bacteria. Annu. Rev. Microbiol. 55: 165-199.

Nasser W and Reverchon S (2007). New insights into the regulatory mechanisms of the LuxR family of quorum sensing regulators. Anal. Bioanal. Chem. 387: 381-390.

Nealson KH and Hastings JW (1979). Bacterial bioluminescence: its control and ecological significance. Microbiol. Rev. 43: 496-518.

Nylander JAA (2004). MrModeltest v2. Program distributed by the author. Evolutionary Biology Centre, Uppsala University, Sweden.

Ravn L, Christensen AB, Molin S, Givskov M, et al. (2001). Methods for detecting acylated homoserine lactones produced by Gram-negative bacteria and their application in studies of AHL-production kinetics. J. Microbiol. Methods. 44: 239-251.

Sabag-Daigle A, Soares JA, Smith JN, Elmasry ME, et al. (2012). The acyl homoserine lactone receptor, SdiA, of Escherichia coli and Salmonella enterica serovar Typhimurium does not respond to indole. Appl. Environ. Microbiol. 78: 5424-5431.

Shaw PD, Ping G, Daly SL, Cha C, et al. (1997). Detecting and characterizing N-acyl-homoserine lactone signal molecules by thin-layer chromatography. Proc. Natl. Acad. Sci. U. S. A. 94: 6036-6041.

Sitnikov DM, Schineller JB and Baldwin TO (1996). Control of cell division in Escherichia coli: regulation of transcription of ftsQA involves both rpoS and SdiA-mediated autoinduction. Proc. Natl. Acad. Sci. U. S. A. 93: 336-341.

Smith JN, Dyszel JL, Soares JA, Ellermeier CD, et al. (2008). SdiA, an $N$-Acylhomoserine lactone receptor, becomes active during the transit of Salmonella enterica through the gastrointestinal tract of turtles. PLOS One. 7: e2826.

Sockett PN (1991). A review: The economic implications of human Salmonella infection. J. Appl. Bacteriol. 71: 289-295.

Swift S, Downie JA, Whitehead NA, Barnard AM, et al. (2001). Quorum sensing as a population-density-dependent determinant of bacterial physiology. Adv. Microb. Physiol. 45: 199-270.

Taga ME, Semmelhack JL and Bassler BL (2001). The LuxS-dependent autoinducer AI-2 controls the expression of an ABC transporter that functions in AI-2 uptake in Salmonella Typhimurium. Mol. Microbiol. 42: 777-793.

Tamura K, Peterson D, Peterson N, Stecher G, et al. (2011). MEGA 5: molecular evolutionary genetics analysis using maximum likelihood, evolutionary distance, and maximum parsimony methods. Mol. Biol. Evol. 28: 2731-2739.

Thomson NR, Clayton DJ, Windhorst D, Vernikos G, et al. (2008). Comparative genome analysis of Salmonella Enteritidis PT4 and Salmonella Gallinarum 287/91 provides insights into evolutionary and host adaptation pathways. Genome Res. 18: 1624-1637.

Walters M and SperadnioV (2006). Quorum sensing in Escherichia coli and Salmonella. Int. J. of Med. Microbiol. 296: $125-131$.

Yang Z and Rannala B (1997). Bayesian phylogenetic inference using DNA sequences: a Markov Chain Monte Carlo method. Mol. Biol. Evol. 14: 717-724. 